Provided for non-commercial research and education use. Not for reproduction, distribution or commercial use.

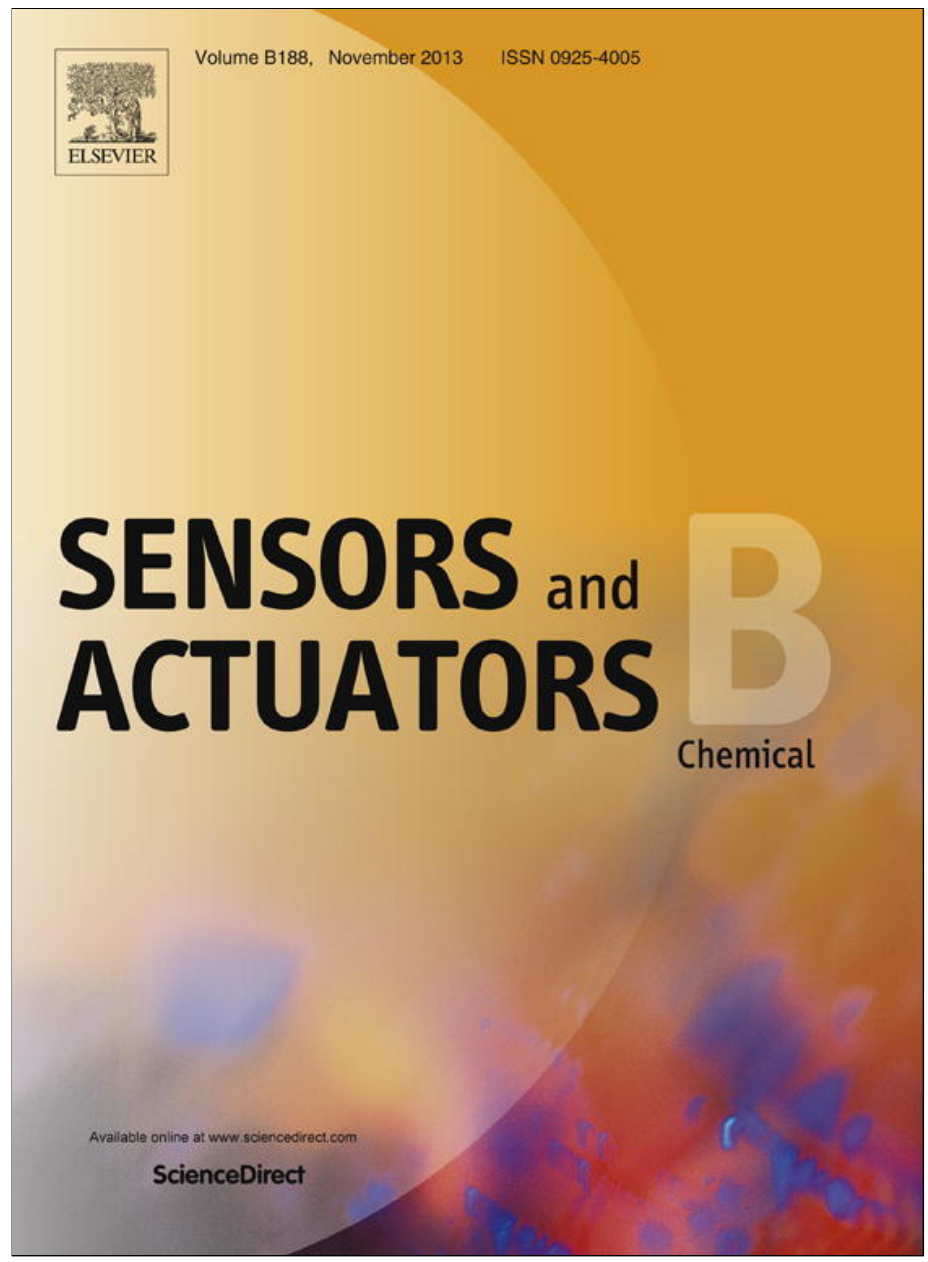

This article appeared in a journal published by Elsevier. The attached copy is furnished to the author for internal non-commercial research and education use, including for instruction at the authors institution and sharing with colleagues.

Other uses, including reproduction and distribution, or selling or licensing copies, or posting to personal, institutional or third party websites are prohibited.

In most cases authors are permitted to post their version of the article (e.g. in Word or Tex form) to their personal website or institutional repository. Authors requiring further information regarding Elsevier's archiving and manuscript policies are encouraged to visit:

http://www.elsevier.com/authorsrights 


\title{
Molecularly imprinted nanoparticles based potentiometric sensor with a nanomolar detection limit
}

\author{
Rongning Lianga,b, Lijuan Kou ${ }^{\mathrm{a}, \mathrm{b}}$, Zhaopeng Chen ${ }^{\mathrm{a}}$, Wei Qin ${ }^{\mathrm{a}, *}$ \\ ${ }^{a}$ Key Laboratory of Coastal Zone Environmental Processes and Ecological Remediation, Yantai Institute of Coastal Zone Research (YIC), Chinese Academy of \\ Sciences (CAS), Shandong Provincial Key Laboratory of Coastal Zone Environmental Processes, YICCAS, Yantai, Shandong 264003, PR China \\ ${ }^{\mathrm{b}}$ University of Chinese Academy of Sciences, Beijing 100049, PR China
}

\section{A R T I C L E I N F O}

\section{Article history:}

Received 22 May 2013

Received in revised form 27 July 2013

Accepted 29 July 2013

Available online 7 August 2013

\section{Keywords:}

Potentiometric sensors

Molecularly imprinted nanoparticles

Asymmetric membranes

Triclosan

Trace-level analysis

\begin{abstract}
A B S T R A C T
A novel molecularly imprinted nanoparticles based potentiometric sensor with a nanomolar detection limit has been described. Uniform-sized molecularly imprinted nanoparticles are utilized as a recognition receptor to provide a higher affinity since they have a larger binding capability and can be well dispersed in the polymeric membrane of an ion-selective electrode (ISE). The asymmetric membrane rotating ISE configuration is introduced to improve the detection sensitivity by enhancing the nonequilibrium steadystate potential response at the sample-membrane interface. It has been found that the proposed electrode shows a remarkably improved sensitivity over conventional potentiometric sensors based on molecularly imprinted polymers (MIPs). By using triclosan as a model, the new concept offers a low detection limit of $1.9 \times 10^{-9} \mathrm{~mol} \mathrm{~L}^{-1}$ in $0.2 \mathrm{M} \mathrm{NaHCO}_{3} / \mathrm{Na}_{2} \mathrm{CO}_{3}$ of $\mathrm{pH} 10.1$, which is at least two orders of magnitude lower than those of conventional MIPs based potentiometric sensors. The practical application of the proposed sensor has been carried out for the detection of trace levels of triclosan in toothpaste samples.
\end{abstract}

(c) 2013 Elsevier B.V. All rights reserved.

\section{Introduction}

In recent years, molecularly imprinted polymers (MIPs) have gained wide acceptance as new artificial recognition receptors in chemical sensors since they have high affinities and excellent selectivities similar to those of antibodies, and are chemically stable and easy to produce [1-3]. Various electrochemical sensors have been fabricated using MIPs as receptors, based on capacitance [4], conductometry [5], amperometry [6], voltammetry [7], quartz microbalance [8] and impedance spectroscopy [9]. As generic and highly successful approaches to chemical sensing, potentiometric sensors have shown to be very promising for trace-level environmental and biological measurements $[10,11]$. Nowadays, potentiometric sensors based on MIPs have been successfully developed for determination of anions, cations and neutral species [12-16]. However, it should be noted that most of the MIPs based potentiometric sensors developed so far have poor detection limits in the micromolar range, which are unsuitable for trace-level measurements, especially with high levels of electrolyte background.

Molecularly imprinted nanoparticles have attracted considerable attention in recent years because they possess many remarkable advantages over conventional imprinting materials such as high surface-to-volume ratios, large binding capabilities,

\footnotetext{
* Corresponding author. Tel.: +86 535 2109156; fax: +86 5352109000.

E-mail address: wqin@yic.ac.cn (W. Qin).
}

fast binding kinetics and ease of use with standard liquid dispensers [17-20]. Such characteristics make MIP nanoparticles suitable for construction of electrochemical sensors [9]. However, to date, very few of the nano-imprinted materials have been exploited in polymeric membrane potentiometric sensors [21].

Recently, we constructed an asymmetric membrane rotating ion-selective electrode (ISE) configuration to significantly improve the detection sensitivity of potentiometric measurements in the presence of a high-interfering background [22]. The response mechanism of the proposed ISE sensing mode is based on the nonequilibrium steady-state diffusion process of primary ions at the sample-membrane interface [23-25]. This promising protocol has been demonstrated to exhibit a subnanomolar detection limit using a commercially available ionophore (e.g. ETH 1062).

The aim of this work is to combine the unique properties of imprinted nanomaterials with the asymmetric membrane rotating ISE configuration to fabricate MIP based potentiometric sensor for trace-level analysis with a high-interfering background. For this purpose, an asymmetric polymeric membrane ISE configuration based on MIP nanobeads as a receptor is prepared. MIP nanobeads are used to provide an excellent affinity for the target molecules and the asymmetric membrane rotating ISE configuration is utilized to improve the detection sensitivity. A conditioning solution containing only interfering ions is applied to eliminate the ion-exchange effect [22]. The new concept has been evaluated and illustrated for the trace-level detection of triclosan as an example of high practical importance. Triclosan is an antimicrobial agent used in a variety of 


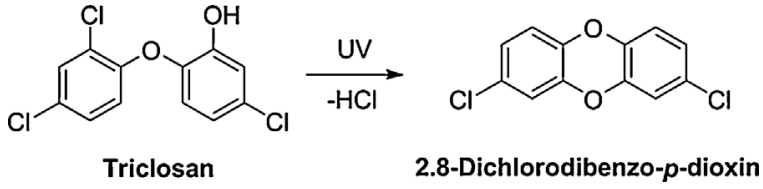

Scheme 1. Photochemical conversion of triclosan to 2,8-dichlorodibenzo-p-dioxin.

consumer products but can act as a potent inhibitor of enzyme [26] and undergo cyclization to form 2,8-dichlorodibenzo-p-dioxin (2,8DCDD) upon exposure to UV radiation (Scheme 1) [27]. Triclosan is a weak acid with a $\mathrm{p} K_{\mathrm{a}}$ of 7.8, and is readily deprotonated in an aqueous solution at $\mathrm{pH}$ levels higher than 7.8. The deprotonated triclosan can be measured potentiometrically by the proposed ISE. This paper shows that the present asymmetric nano-MIP membrane based ISE can offer a remarkably improved sensitivity for measuring triclosan in the presence of a high-interfering background.

\section{Experimental}

\subsection{Reagents and materials}

Triclosan, acrylamide (AA), methyl methacrylate (MMA), trimethylolpropane trimethacrylate (TRIM), divinylbenzene 80 (DVB 80), acetonitrile and 2,2'-azobisisobutyronitrle (AIBN) were purchased from Aldrich. High molecular weight poly (vinyl chloride) (PVC), 2-nitrophenyl octyl ether (o-NPOE) and tridodecylmethylammonium chloride (TDMAC) were obtained from Fluka AG (Buchs, Switzerland). Tetrahydrofuran (THF) was freshly distilled and AA was recrystallized from methanol prior to use. All other reagents were analytical grade and used without any further purification. Aqueous solutions were prepared with freshly deionized water (18.2 M $\Omega \mathrm{cm}$ specific resistance) obtained with a Pall Cascada laboratory water system. A stock solution of $0.01 \mathrm{M}$ triclosan was prepared by dissolving $28.9 \mathrm{mg}$ of triclosan in $10 \mathrm{~mL}$ of acetonitrile and then diluted to various concentrations of working solutions with $0.2 \mathrm{M} \mathrm{NaHCO} / \mathrm{Na}_{2} \mathrm{CO}_{3}$ of $\mathrm{pH} 10.1$.

\subsection{Synthesis of triclosan MIP}

The triclosan MIP nanobeads were synthesized by the precipitation method as described elsewhere [28,29]. Briefly, the template triclosan (1.0 mmol), AA (2.5 mmol), MMA (4.0 mmol), DVB $(60 \mu \mathrm{L})$, TRIM $(320 \mu \mathrm{L})$ and free-radical initiator AIBN $(0.5 \mathrm{mmol})$ were dissolved in acetonitrile $(40 \mathrm{~mL})$ in a $50 \mathrm{~mL}$ flask and sonicated for $10 \mathrm{~min}$ to maintain homogeneity. Then the solution was purged with gentle flow of $\mathrm{N}_{2}$ for $10 \mathrm{~min}$ and sealed under $\mathrm{N}_{2}$ atmosphere. Polymerization was carried out by submerging the flask in a water bath and rotating the flask at a speed of $10 \mathrm{rpm}$. The temperature was increased from $20^{\circ} \mathrm{C}$ to $75^{\circ} \mathrm{C}$ within $30 \mathrm{~min}$ and maintained at $75^{\circ} \mathrm{C}$ for $24 \mathrm{~h}$. After polymerization, the template was removed by the batch-mode solvent extractions with methanol/acetic acid $(8 / 2, v / v)$ and methanol until no absorption of the methanol at $280 \mathrm{~nm}$ was observed with a Beckman DU-800 UV spectrophotometer. The resulting polymer was dried for $10 \mathrm{~h}$ in vacuum at $50^{\circ} \mathrm{C}$. Non-imprinted polymer (NIP) was prepared under identical conditions except for omission of the template. Traditional MIP microbeads were prepared according to the precipitation method described before [16].

\subsection{Preparation of polymeric membranes and ISES}

The homogeneous ISE membranes based on triclosan MIP contained (in wt\%) MIP or NIP (7.6), TDMAC (1.5), o-NPOE (60.6), and PVC (30.3). The components of each membrane (totaling $360 \mathrm{mg}$ ) were dissolved in THF (3.5 mL) and poured into a glass ring (i.d. $36 \mathrm{~mm}$ ) fixed on a glass plate. Overnight evaporation of the solvent yielded membranes of $\sim 200-\mu \mathrm{m}$ thickness. For each electrode, a disk of $8-\mathrm{mm}$ diameter was punched from the membranes and glued to a plasticized PVC tubing with THF/PVC slurry. The asymmetric MIP membranes were prepared by evenly spreading $5 \mu \mathrm{L}$ of a TDMAC solution $(0.7 \mathrm{mg} / \mathrm{mL}$ in THF) on the surface ( 8 - $\mathrm{mm}$ diameter) of the hard membrane matrix containing (in wt\%) MIP (7.6), ETH 500 (1.0), o-NPOE (45.7), and PVC (45.7) and letting the active layer dry for $1 \mathrm{~h} .0 .2 \mathrm{M} \mathrm{NaHCO}_{3} / \mathrm{Na}_{2} \mathrm{CO}_{3}$ of $\mathrm{pH} 10.1$ and $0.01 \mathrm{M} \mathrm{NaCl}$ were used as the internal filling solutions for measurement of triclosan and for evaluation of the selectivity coefficients, respectively. All the electrodes were conditioned in a solution identical to the inner filling solution for 1 day.

\subsection{EMF measurements}

For measurements with the rotating disk ISE, a rotating silver disk electrode (ATA-1B, Jiangsu Jiangfen Electroanalytical Instrument Co., Ltd., China) with a diameter of $3 \mathrm{~mm}$ was used as the internal reference electrode. The silver disk electrode was chloridized with a solution of $1 \mathrm{M} \mathrm{HCl}$ containing $0.1 \mathrm{M} \mathrm{FeCl}_{3}$. The plasticized PVC tubing with the asymmetric MIP based ISE membrane glued at the distal end was filled with the internal filling solution containing $0.01 \mathrm{M} \mathrm{NaCl}$ in $0.2 \mathrm{M}$ carbonate buffer of $\mathrm{pH}$ 10.1 and then screwed onto the silver disk electrode. The external reference electrode employed was a double-junction saturated calomel electrode (SCE) with 0.1 M LiOAc as the bridge electrolyte. All measurements were performed with a rotating (at $3000 \mathrm{rpm}$ ) or static mode configuration (using a stir bar operating at $200 \mathrm{rpm}$, and an $\mathrm{Ag} / \mathrm{AgCl}(3 \mathrm{M} \mathrm{KCl})$ inner reference electrode) to achieve solution-phase convection. Electromotive force (EMF) values were measured at $20-21^{\circ} \mathrm{C}$ using a PXSJ-216 pH meter (Shanghai, China).

Selectivity coefficients were determined by the separate solution method [30]. The EMF values for inorganic ions were corrected for the liquid-junction potentials with the Henderson equation. The ion activity coefficients were calculated according to the Debye-Hückel equation.

\section{Results and discussion}

\subsection{Characterization of MIP nanobeads}

The morphologies of the obtained triclosan MIP nanobeads were characterized by scanning electron microscopy (SEM). As shown in Fig. 1a, the triclosan imprinted nanobeads are uniform and spherical with a diameter distribution of $200-300 \mathrm{~nm}$. These uniform-sized nanobeads can be well dispersed in the polymeric ISE membrane, which could induce more binding sites available in the membrane and a lower membrane impedance [16], as compared to traditional MIP microbeads with a diameter of ca. $2 \mu \mathrm{m}$ (Fig. 1c). The SEM images also indicate the NIP nanobeads prepared with the same recipe have the similar morphological structure and particle size distribution (Fig. 1b).

\subsection{Optimization of the proposed sensor}

To illustrate the excellent binding affinity of triclosan MIP nanobeads, two kinds of ISEs based on MIPs were firstly compared: one with MIP nanobeads as ionophore and the other with traditional MIP microbeads. As illustrated in Fig. 2, the electrode prepared with the MIP nanobeads (curve a) shows a better performance than that obtained with the MIP microbeads (curve b). For measurement of triclosan at $10^{-4} \mathrm{M}$ in the presence of $0.2 \mathrm{M}$ $\mathrm{NaHCO}_{3} / \mathrm{Na}_{2} \mathrm{CO}_{3}$, the EMF change obtained by the MIP nanobeads 


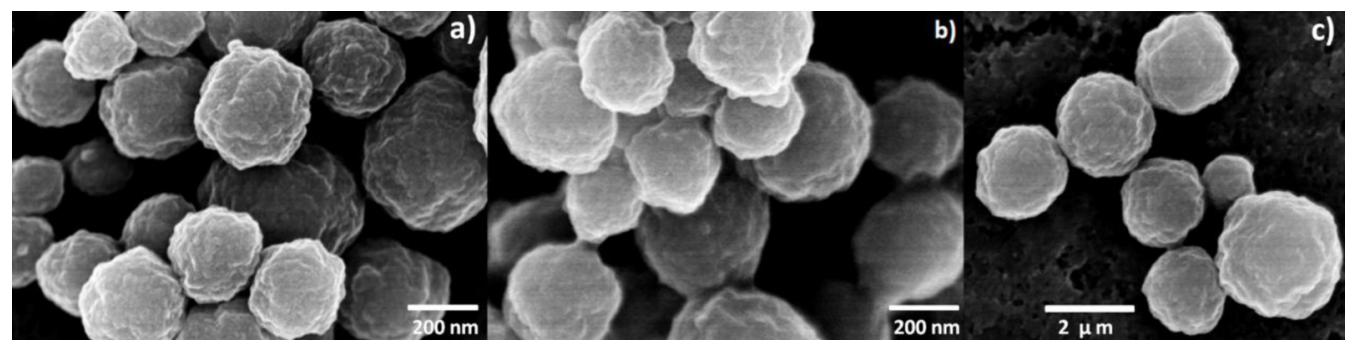

Fig. 1. SEM images of the triclosan MIP nanobeads (a), NIP nanobeads (b) and MIP microbeads (c).

based ISE is approximately $255 \mathrm{mV}$, while that by the electrode with MIP microbeads as ionophore is $190 \mathrm{mV}$. In addition, the membrane ISE prepared with the MIP nanobeads shows a shorter response time ( $\sim 3 \mathrm{~min}$ ), defined as the time required to achieve $95 \%$ of the stable signal, than that with MIP microbeads ( $\sim 5 \mathrm{~min}$ ). These observations might be attributed to the fact that uniform nanobeads have a higher binding capability and can be well dispersed in the polymeric ISE membrane, thus leading to more available binding sites in the membrane [16]. For a comparison, the potential response of the traditional homogeneous membrane conditioned with the primary ion was tested. The results are shown in Figure S1 in the Supplementary Materials. As can be seen, the traditional membrane electrode has a rather poor detection limit of $3.9 \times 10^{-6} \mathrm{M}(3 \sigma)$ in $0.2 \mathrm{M}$ carbonate buffer of $\mathrm{pH} 10.1$, which is due to the leaching of the primary ions from the sensing membrane into the sample solution [31-35]. As a control, the potential response of NIP nanobeads based ISE membrane was also investigated. Compared with the response of MIP nanobeads based ISE, neglectable EMF changes below $10^{-5} \mathrm{M}$ triclosan can be observed for NIP based membrane (curve c), which indicates the MIP is effective for specific recognition of the target ion.

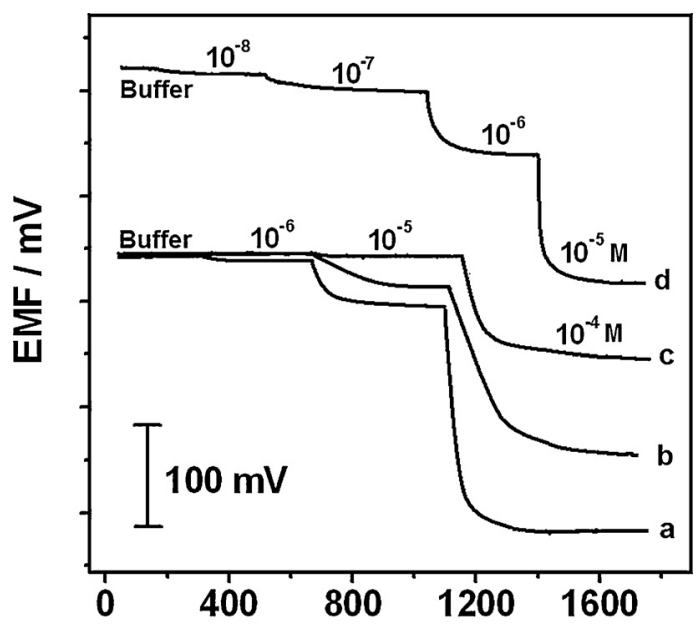

Time / s

Fig. 2. Potential response curves obtained by ISEs with the homogeneous membranes containing MIP nanobeads (curve a), MIP microbeads (curve b) and NIP nanobeads (curve c) under constant stirring, and by the asymmetric membrane based on MIP nanobeads with rotation at $3000 \mathrm{rpm}$ (curve d). Nano-MIP membrane (curve a): $7.6 \mathrm{wt} \%$ MIP nanobeads, $1.5 \mathrm{wt} \%$ TDMAC, $60.6 \mathrm{wt} \%$ o-NPOE and $30.3 \mathrm{wt} \%$ PVC; micro-MIP membrane (curve b): 7.6 wt\% MIP microbeads, 1.5 wt\% TDMAC, $60.6 \mathrm{wt} \%$ o-NPOE and $30.3 \mathrm{wt} \%$ PVC; nano-NIP membrane (curve c): $7.6 \mathrm{wt} \% \mathrm{NIP}$ nanobeads, $1.5 \mathrm{wt} \%$ TDMAC, $60.6 \mathrm{wt} \% \mathrm{o}$-NPOE and $30.3 \mathrm{wt} \%$ PVC. The asymmetric nano-MIP membrane (curve d) was formed by evenly spreading $5 \mu \mathrm{L}$ of a TDMAC solution $(0.7 \mathrm{mg} / \mathrm{mL}$ in THF) on the surface of the membrane matrix containing $7.6 \mathrm{wt} \%$ MIP nanobeads, $1.0 \mathrm{wt} \%$ ETH 500, $45.7 \mathrm{wt} \%$ o-NPOE, and $45.7 \mathrm{wt} \%$ PVC. All the measurements were performed in $0.2 \mathrm{M}$ carbonate buffer of $\mathrm{pH} 10.1$.
As shown above, the detection sensitivity of the MIP based ISE can be improved by using MIP nanobeads as ionophore. However, the obtained detection limit cannot satisfy the requirement for trace-level analysis. The asymmetric membrane rotating ISE configuration was then employed for further improvement of the detection sensitivity by promoting the accumulation of the primary ions in the boundary layer of the membrane surface. The asymmetric nano-MIP membrane was formed by evenly spreading the ion-exchanger solution onto the surface of PVC membrane as described before [22]. The illustration of MIP based asymmetric ISE membrane is shown in Scheme 2. The detection mechanism of the proposed asymmetric membrane has been described before [22]. Unlike the traditional ISEs, the membrane electrode based on MIP particles is conditioned in a solution of interfering ions (e.g. $\mathrm{HCO}_{3}{ }^{-}$and $\mathrm{CO}_{3}{ }^{2-}$ ) instead of primary ions so that the membrane is completely occupied by $\mathrm{HCO}_{3}{ }^{-}$and $\mathrm{CO}_{3}{ }^{2-}$ and no primary ions (deprotonated triclosan) exist in the ISE membrane. When the electrode is in contact with the deprotonated triclosan, the interfering ions in the membrane surface can be partially displaced by the deprotonated triclosan due to the favorable host-guest interaction with the MIP nanoparticles in the membrane, thus causing a steady-state potential response [23]. Since the EMF response of the proposed discriminated-ion-conditioned electrode is highly dependent on the accumulation of the deprotonated triclosan in the interfacial layer of the membrane phase, the detection sensitivity can be largely improved by accelerating ion fluxes in the aqueous Nernst layer and/or suppressing ion fluxes from the phase boundary into the bulk of the membrane [22]. As shown in Scheme 2, by using the asymmetric membrane with an ion-exchanger (e.g. TDMAC) loaded only on the membrane surface, the diffusion of the deprotonated triclosan from the organic boundary layer into the bulk of the membrane can be effectively blocked, while rotation of the membrane electrode dramatically reduces the diffusion layer thickness of aqueous phase and significantly promotes the mass

\section{MIP based asymmetric ISE membrane}

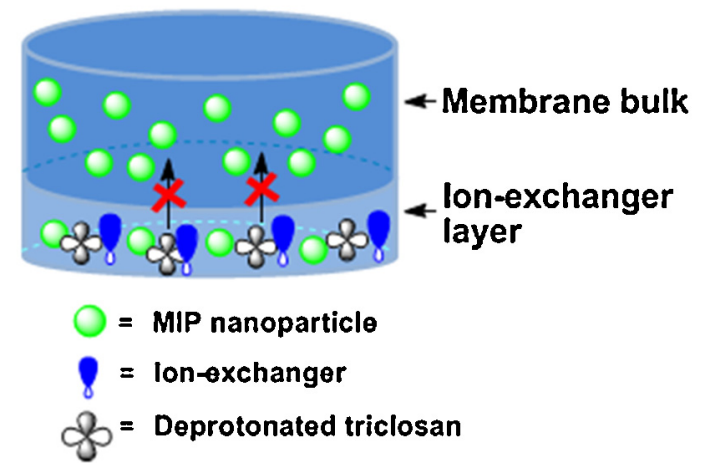

Scheme 2. Illustration of the MIP based asymmetric ISE membrane. 


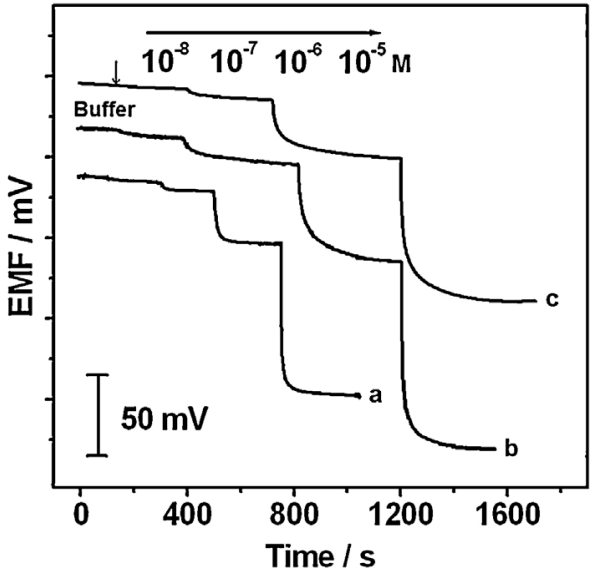

Fig. 3. Potentiometric responses of the asymmetric membrane based ISEs prepared with the membranes possessing different PVC/o-NPOE ratios of 2.5:1 (curve a), 1:1 (curve b), and 1:2 (curve c). The other conditions are as given in Fig. 2 (curve d).

transfer of the deprotonated triclosan to the sample-membrane interface [36,37]. The induced accumulation of the deprotonated triclosan in the membrane boundary layer largely enhances the obtained non-equilibrium potential response.

In order to decrease the resistance of the PVC membrane bulk which lacks of ion-exchanger, the inert lipophilic electrolyte ETH 500 was incorporated into the asymmetric ISE membrane [38]. Curve $d$ in Fig. 2 shows the potential response associated with the asymmetric membrane rotating ISE based on MIP nanobeads. As expected, the asymmetric membrane shows a significantly improved performance over the conventional homogeneous membrane based electrode. A negligible EMF change was observed using the homogeneous membrane electrode for $10^{-6} \mathrm{M}$ triclosan, while that by the asymmetric membrane electrode was approximately $85 \mathrm{mV}$. It can be demonstrated that the asymmetric membrane rotating ISE configuration is effective for the sensitivity improvement of the MIP based ISE.

The experimental parameters were further examined for optimum analytical performance. Since using a hard polymeric membrane (with high PVC content) can improve the detection sensitivity by enhancing the accumulation of primary ions in the surface boundary layer of the membrane [22,23], the effect of $\mathrm{PVC} /$ plasticizer ratio on the potential responses of asymmetric membrane electrode was tested, and the results are illustrated in Fig. 3. It can be seen that the total EMF change from $10^{-5}$ to $10^{-8} \mathrm{~mol} \mathrm{~L}^{-1}$ increases with decrease in the plasticizer content, which is attributed to the fact that a lower plasticizer content leads to a smaller diffusion coefficient, thus accelerating the accumulation of deprotonated triclosan at the boundary layer of the MIP membrane. However, further decrease in the plasticizer content may result in a smaller potential response, which is probably due to the poor dispersion of MIP nanobeads in the polymeric membrane with a high PVC content. On the other hand, it should be noted that a lower plasticizer content may lead to a higher membrane resistance as well as a longer conditioning time [39]. Therefore, the $\mathrm{PVC} /$ plasticizer ratio of $1: 1$ with a maximum response was chosen for the proposed membrane. The rotation speed could induce a large influence on the sensitivity of a potentiometric sensor with a nonequilibrium steady-state response $[24,40]$. As shown in Fig. 4, the potential response increases with increase in rotation speed. However, experiments also showed that larger noise levels in the potential response could be observed when the rotation speeds were higher than $3000 \mathrm{rpm}$ [22]. Therefore, $3000 \mathrm{rpm}$ was selected as the optimal rotation speed for the present sensor.

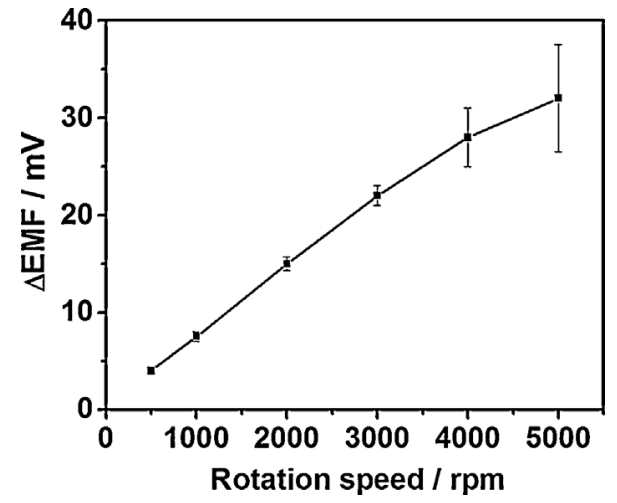

Fig. 4. Effect of rotation speed on the potentiometric response of the asymmetric nano-MIP membrane based ISE to $10^{-7} \mathrm{M}$ triclosan. Each error bar represents one standard deviation for six measurements. The other conditions are as given in Fig. 2 (curve d).

\subsection{Characteristics of the proposed sensor}

Under the optimized conditions, the calibration curve of the asymmetric membrane rotating ISE configuration based on MIP nanobeads as ionophore was obtained. Fig. 5 shows the potential response of the proposed electrode for measuring triclosan at concentrations ranging from $10^{-5}$ to $10^{-8} \mathrm{~mol} \mathrm{~L}^{-1}$ in a $0.2 \mathrm{M}$ $\mathrm{NaHCO}_{3} / \mathrm{Na}_{2} \mathrm{CO}_{3}$ buffer of $\mathrm{pH} 10.1$. As can be seen, there is a linear dependence of the EMF change on the concentration of triclosan in the range of $1.0 \times 10^{-8}$ to $1.0 \times 10^{-6} \mathrm{~mol} \mathrm{~L}^{-1}(\gamma=0.999)$ with a detection limit of $1.9 \times 10^{-9} \mathrm{~mol} \mathrm{~L}^{-1}(3 \sigma)$. This detection limit is at least two orders of magnitude lower than those of most of the MIPs based potentiometric sensors [12], which makes it feasible to perform trace-level measurements in environmental samples.

After each measurement, the asymmetric membrane ISE was washed with $20 \mathrm{~mL}$ of an aqueous solution containing $20 \mathrm{wt} \%$ ethanol and $1 \mathrm{wt} \%$ acetic acid for $30 \mathrm{~s}$ to remove triclosan in the MIP and then immersed in the $\mathrm{NaHCO}_{3} / \mathrm{Na}_{2} \mathrm{CO}_{3}$ buffer to obtain the original baseline potential value. For ten consecutive measurements, the standard deviation of the potential responses to $10^{-7} \mathrm{M}$ triclosan was $1.3 \mathrm{mV}$, which implies that the lipophilic electrolyte and the plasticizer cannot largely be removed from the membrane during the regeneration steps. The diffusion of the ion-exchanger from the membrane surface layer into the membrane bulk would occur, which may change the composition of the active layer. The potential stability of the asymmetric membrane was tested and

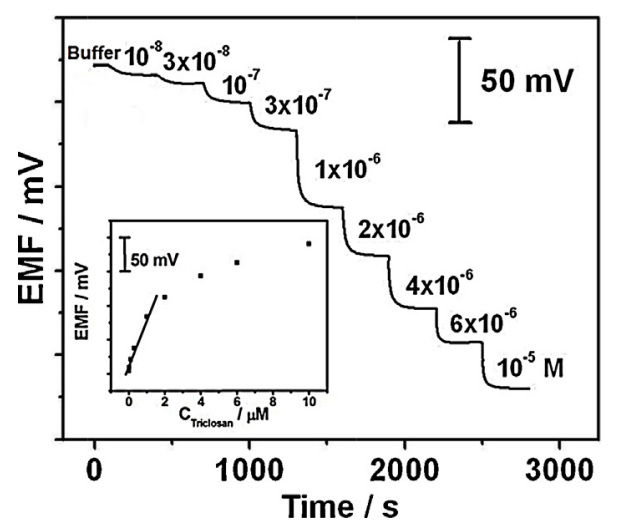

Fig. 5. Time-dependent EMF response traces of the asymmetric nano-MIP membrane rotating electrode for measuring deprotonated triclosan. The inset shows the corresponding calibration curve. The other conditions are as given in Fig. 2 (curve d). 
Table 1

Potentiometric selectivity coefficients, $\log K_{\mathrm{M}, \mathrm{X}}^{\mathrm{pot}}$, of the proposed asymmetric nano-MIP membrane ISE ${ }^{\mathrm{a}}$.

\begin{tabular}{llll}
\hline Interfering ion, $\mathrm{X}^{\mathrm{b}}$ & $\log K_{\mathrm{M}, \mathrm{X}}^{\mathrm{pot}}$ & Interfering ion, $\mathrm{X}$ & $\log K_{\mathrm{M}, \mathrm{X}}^{\mathrm{pot}}$ \\
\hline 4-Phenoxyphenol & $-9.7 \pm 1.1(8.5 \pm 0.8)^{\mathrm{c}}$ & $\mathrm{HCO}_{3}{ }^{-}$ & $-9.5 \pm 0.9$ \\
4-Methoxyphenol & $-2.2 \pm 0.2$ & $\mathrm{CO}_{3}{ }^{-}$ & $-7.4 \pm 0.8$ \\
Catechol $^{-}$ & $-4.3 \pm 0.4$ & $\mathrm{OH}^{-}$ & $-5.8 \pm 0.2$ \\
$\mathrm{Cl}^{-}$ & $-3.9 \pm 0.3$ & $\mathrm{NO}_{3}{ }^{-}$ & $-7.9 \pm 0.5$ \\
\hline
\end{tabular}

a Mean value obtained from three corresponding pairs of concentrations of deprotonated triclosan and the respective interfering anion in the Nernstian response range \pm SD

b The sample $\mathrm{pH}$ was adjusted to 12.0 in order to deprotonate the organic substance by using $\mathrm{NaOH}$.

c Value in the parentheses is the selectivity coefficient for the micro-MIP membrane based ISE.

Table 2

Application of the proposed method to determination of triclosan in toothpaste samples spiked with different amounts of triclosan.

\begin{tabular}{lccc}
\hline Sample & \multicolumn{2}{l}{ Amounts of triclosan $\left(\mu \mathrm{g} \mathrm{g}^{-1}\right)$} & \multirow{2}{*}{ Recovery (\%) } \\
\cline { 2 - 3 } & Amount added & Amount found ${ }^{\mathrm{a}}$ & \\
\hline 1 & 5.0 & $4.8 \pm 0.5$ & 96 \\
2 & 20.0 & $21.0 \pm 1.5$ & 105 \\
3 & 100.0 & $102.1 \pm 8.7$ & 102 \\
4 & 300.0 & $319.1 \pm 15.7$ & 106 \\
\hline
\end{tabular}

${ }^{a}$ Average value of three determinations \pm standard deviation.

the results showed that no significant change in sensitivity was observed after storage of the membrane at $4{ }^{\circ} \mathrm{C}$ for two weeks.

\subsection{Interference study}

The selectivity of the proposed asymmetric membrane was characterized by using Bakker' method to eliminate the influence of the inherent sensitivity limit on the ISE response toward interfering ions [30]. The logarithmic Nikolskii coefficients for triclosan $\left(\log K_{\mathrm{M}, \mathrm{X}}^{\mathrm{pot}}\right)$ over other related structure analogs and discriminated ions are summarized in Table 1. It can be seen that the proposed electrode shows excellent selectivity to triclosan over its analogs such as 4-phenoxyphenol, 4-methoxyphenol and catechol and some inorganic anions. Notably, the obtained logarithmic selectivity coefficients of the asymmetric nano-MIP membrane based ISE over interfering ions such as $\mathrm{HCO}_{3}{ }^{-}$are superior to those of the micro-MIP membrane based ISE, which further confirms that MIP nanobeads have a higher affinity than microbeads. The high sensitivity of the proposed asymmetric membrane rotating electrode offers great potential for trace-level potentiometric detection.

\subsection{Application}

The proposed asymmetric nano-MIP membrane based ISE exhibits excellent affinity and high sensitivity for the determination of trace-level triclosan under optimum experimental conditions. In order to illustrate its accuracy in practical analysis, the proposed electrode was applied to analyze triclosan in spiked toothpaste samples. A portion of the toothpaste was weighted and dissolved in $0.2 \mathrm{M} \mathrm{NaHCO}_{3} / \mathrm{Na}_{2} \mathrm{CO}_{3}$ buffer solution. After filtering, the triclosan contents in toothpaste were potentiometrically analyzed with the proposed ISE. The results are given in Table 2. It can be seen that the recoveries of toothpaste samples vary from $96 \%$ to $106 \%$, indicating that the proposed potentiometric sensor has promising feasibility for trace-level analysis of triclosan in complex samples.

\section{Conclusions}

A novel asymmetric polymeric membrane ISE using MIP nanobeads as ionophore for the determination of trace-level triclosan has been described, based on the combination of the excellent affinity of the nano-MIP beads and high sensitivity of the asymmetric membrane rotating ISE configuration. The proposed electrode has shown remarkably improved sensitivity for triclosan detection with a detection limit of $1.9 \times 10^{-9} \mathrm{~mol} \mathrm{~L}^{-1}$. Since many MIPs have been extensively exploited in analytical chemistry, this methodology is promising to develop MIP based potentiometric sensors for trace-level measurements of various ionic species.

\section{Acknowledgements}

This work was financially supported by the Instrument Developing Project of the Chinese Academy of Sciences (YZ201161), the National Natural Science Foundation of China (41206087, 20977073), the Department of Science and Technology of Shandong Province (BS2009DX006) and the Taishan Scholar Program of Shandong Province (TS20081159).

\section{Appendix A. Supplementary data}

Supplementary material related to this article can be found, in the online version, at http://dx.doi.org/10.1016/j.snb.2013.07.110.

\section{References}

[1] L.X. Chen, S.F. Xu, J.H. Li, Recent advances in molecular imprinting technology: current status, challenges and highlighted applications, Chemical Society Reviews 40 (2011) 2922-2942.

[2] S. Banerjee, B. König, Molecular imprinting of luminescent vesicles, Journal of the American Chemical Society 135 (2013) 2967-2970.

[3] Y. Fuchs, O. Soppera, A.G. Mayes, K. Haupt, Holographic molecularly imprinted polymers for label-free chemical sensing, Advanced Materials 25 (2013) 566-570

[4] T. Alizadeh, A. Akbari, A capacitive biosensor for ultra-trace level urea determination based on nano-sized urea-imprinted polymer receptors coated on graphite electrode surface, Biosensors and Bioelectronics 43 (2013) 321-327.

[5] C.Y. Chai, G.Y. Liu, F. Li, X.F. Liu, B. Yao, L. Wang, Towards the development of a portable sensor based on a molecularly imprinted membrane for the rapid determination of salbutamol in pig urine, Analytica Chimica Acta 675 (2010) $185-190$.

[6] J. Zhang, Y.Q. Wang, R.H. Lv, L. Xu, Electrochemical tolazoline sensor based on gold nanoparticles and imprinted poly-o-aminothiophenol film, Electrochimica Acta 55 (2010) 4039-4044

[7] A. Gómez-Caballero, N. Unceta, M.A. Goicolea, R.J. Barrio, Voltammetric determination of metamitron with an electrogenerated molecularly imprinted polymer microsensor, Electroanalysis 19 (2007) 356-363.

[8] S.P. Pogorelova, T. Bourenko, A.B. Kharitonov, I. Willner, Selective sensing of triazine herbicides in imprinted membranes using ion-sensitive filed-effect transistors and microgravimetric quartz crystal microbalance measurements, Analyst 127 (2002) 1484-1491.

[9] M.C. Blanco-López, M.J. Lobo-Castañón, A.J. Miranda-Ordieres, P. Tuñón-Blanco, Electrochemical sensors based on molecularly imprinted polymers, Trends in Analytical Chemistry 23 (2004) 36-48.

[10] E. Bakker, E. Pretsch, Modern potentiometry, Angewandte Chemie International Edition 46 (2007) 5660-5668

[11] J. Bobacka, A. Ivaska, A. Lewenstam, Potentiometric ion sensors, Chemical Reviews 108 (2008) 329-351.

[12] T.P. Rao, R. Kala, Potentiometric transducer based biomimetic sensors for priority envirotoxic markers - an overview, Talanta 76 (2008) 485-496.

[13] H.M.V. Oliveira, F.T.C. Moreira, M.G.F. Sales, Ciprofloxacin-imprinted polymeric receptors as ionophores for potentiometric transduction, Electrochimica Acta 56 (2011) 2017-2023.

[14] M. Javanbakht, S.E. Fard, A. Mohammadi, M. Abdouss, M.R. Ganjali, P. Norouzi L. Safaraliee, Molecularly imprinted polymer based potentiometric sensor for 
the determination of hydroxyzine in tablets and biological fluids, Analytica Chimica Acta 612 (2008) 65-74.

[15] R.N. Liang, R.M. Zhang, W. Qin, Potentiometric sensor based on molecularly imprinted polymer for determination of melamine in milk, Sensors and Actuators B 141 (2009) 544-550.

[16] R.N. Liang, D.A. Song, R.M. Zhang, W. Qin, Potentiometric sensing of neutral species based on a uniform-sized molecularly imprinted polymer as a receptor, Angewandte Chemie International Edition 49 (2010) 2556-2559.

[17] A. Cumbo, B. Lorber, P.F.X. Corvini, W. Meier, P. Shahgaldian, A synthetic nanomaterial for virus recognition produced by surface imprinting, Nature Communications (2013), http://dx.doi.org/10.1038/ncomms2529.

[18] D.M. Gao, Z.P. Zhang, M.H. Wu, C.G. Xie, G.J. Guan, D.P. Peng, A surface functional monomer-directing strategy for highly dense imprinting of TNT at surface of silica nanoparticles, Journal of the American Chemical Society 129 (2007) 7859-7866.

[19] K. Flavin, M. Resmini, Imprinted nanomaterials: a new class of synthetic receptors, Analytical and Bioanalytical Chemistry 393 (2009) 437-444.

[20] C.G. Xu, L. Ye, Clickable molecularly imprinted nanoparticles, Chemical Communications 47 (2011) 6096-6098.

[21] T. Alizadeh, M.R. Ganjali, M. Akhoundian, Synthesis and application of different nano-sized imprinted polymers for the preparation of promethazine membrane electrodes and comparison of their efficiencies, International Journal of Electrochemical Science 7 (2012) 7655-7674.

[22] W. Qin, R.N. Liang, X.L. Fu, Q.W. Wang, T.J. Yin, W.J. Song, Trace-level potentiometric detection in the presence of a high electrolyte background, Analytical Chemistry 84 (2012) 10509-10513.

[23] B. Fu, E. Bakker, J.H. Yun, V.C. Yang, M.E. Meyerhoff, Response mechanism of polymer membrane-based potentiometric polyion sensors, Analytical Chemistry 66 (1994) 2250-2259.

[24] Q.S. Ye, M.E. Meyerhoff, Rotating electrode potentiometry: lowering the detection limits of nonequilibrium polyion-sensitive membrane electrodes, Analytical Chemistry 73 (2001) 332-336.

[25] M. Maj-Źurawska, T. Sokalski, A. Hulanicki, Interpretation of the selectivity and detection limit of liquid ion-exchanger electrodes, Talanta 35 (1988) 281-286.

[26] C.W. Levy, A. Roujeinikova, S. Sedelnikova, P.J. Baker, A.R. Stuitje, A.R. Slabas, D.W. Rice, J.B. Rafferty, Molecular basis of triclosan activity, Nature 398 (1999) 383-384

[27] J.M. Buth, P.O. Steen, C. Sueper, D. Blumentritt, P.J. Vikesland, W.A. Arnold, K. McNeill, Dioxin photoproducts of triclosan and its chlorinated derivatives in sediment cores, Environmental Science \& Technology 44 (2010) 4545-4551.

[28] K. Yoshimatsu, K. Reimhult, A. Krozer, K. Mosbach, K. Sode, L. Ye, Uniform molecularly imprinted microspheres and nanoparticles prepared by precipitation polymerization: The control of particle size suitable for different analytical applications, Analytica Chimica Acta 584 (2007) 112-121.

[29] L. Ye, R. Weiss, K. Mosbach, Synthesis and characterization of molecularly imprinted microspheres, Macromolecules 33 (2000) 8239-8245.

[30] E. Bakker, Determination of improved selectivity coefficients of polymer membrane ion-selective electrodes by conditioning with a discriminated ion, Journal of the Electrochemical Society 143 (1996) L83-L85.

[31] S. Mathison, E. Bakker, Effect of transmembrane electrolyte diffusion on the detection limit of carrier-based potentiometric ion sensors, Analytical Chemistry 70 (1998) 303-309.
[32] W.E. Morf, M. Badertscher T. Zwickl, N.F de Rooij, E. Pretsch, Effects of ion transport on the potential response of ionophore-based membrane electrodes: a theoretical approach, Journal of Physical Chemistry B 103 (1999) $11346-11356$

[33] T. Zwickl, T. Sokalski, E. Pretsch, Steady-state model calculations predicting the influence of key parameters on the lower detection limit and ruggedness of solvent polymeric membrane ion-selective electrodes, Electroanalysis 11 (1999) 673-680.

[34] T. Sokalski, T. Zwickl, E. Bakker, E. Pretsch, Lowering the detection limit of solvent polymeric ion-selective electrodes. 1. Modeling the influence of steadystate ion fluxes, Analytical Chemistry 71 (1999) 1204-1209.

[35] T. Sokalski, A. Ceresa, M. Fibbioli, T. Zwickl, E. Bakker, E. Pretsch, Lowering the detection limit of solvent polymeric ion-selective membrane electrodes. 2. Influence of composition of sample and internal electrolyte solution, Analytical Chemistry 71 (1999) 1210-1214.

[36] T. Vigassy, R.E. Gyurcsányi, E. Pretsch, Rotating ion-selective membrane electrodes for trace-level measurements, Electroanalysis 15 (2003) 1270-1275.

[37] A. Zirino, R. De Marco, I. Rivera, B. Pejcic, The influence of diffusion fluxes on the detection limit of the jalpaite copper ion-selective electrode, Electroanalysis 14 (2002) 493-498.

[38] J.W. Ding, W. Qin, Current-driven ion fluxes of polymeric membrane ion-selective electrode for potentiometric biosensing, Journal of the American Chemical Society 131 (2009) 14640-14641.

[39] E. Bakker, E. Pretsch, Potentiometric sensors for trace-level analysis, Trends in Analytical Chemistry 24 (2005) 199-207.

[40] W. Qin, W. Zhang, K.P. Xiao, M.E. Meyehoff, Enhanced sensitivity electrochemical assay of low-molecular-weight heparins using rotating polyion-sensitive membrane electrodes, Analytical and Bioanalytical Chemistry 377 (2003) 929-936.

\section{Biographies}

Dr. Rongning Liang received his Doctor's degree in 2011 from Yantai Institute of Coastal Zone Research, Chinese Academy of Sciences. He is now an assistant professor at Yantai Institute of Coastal Zone Research. His current research interests are potentiometric sensors based on molecularly imprinted polymers.

Mrs. Lijuan Kou is a Ph.D. student at Yantai Institute of Coastal Zone Research. She is mainly engaged in the research of potentiometric sensors based on molecularly imprinted polymers.

Dr.Zhaopeng Chen received his Ph.D. in analytical chemistry from Hunan University in 2007. He is now an associate professor at Yantai Institute of Coastal Zone Research. His research interests include nano-analysis and biosensors.

Prof. Wei Oin received his Doctor's degree from Nanjing University in 1998. From 1999 to 2003, he did his postdoctoral research at Swiss Federal Institute of Technology (ETH), University of South Carolina and University of Michigan, respectively. He has been working as Professor at Yantai Institute of Coastal Zone Research since 2006. His research interests are chemical sensors and biosensors for environmental analysis. 